\title{
Emulsion compression and coalescence under enhanced gravity studied with in-situ microscopy
}

\author{
T. Krebs ${ }^{1,2}$, J. J. Slot ${ }^{3}$, C. P. G. H. Schroen ${ }^{2}$, \\ H. W. M. Hoeijmakers ${ }^{3}$ \& R. M. Boom ${ }^{2}$ \\ ${ }^{1}$ Institute for Sustainable Process Technology, The Netherlands \\ ${ }^{2}$ Wageningen University, Food Process Engineering Group, \\ The Netherlands \\ ${ }^{3}$ University of Twente, Engineering Fluid Dynamics Group, \\ The Netherlands
}

\begin{abstract}
We report the results of experiments and numerical calculations of compression and coalescence in a monodisperse oil-in-water emulsion upon centrifugation. A custom-built setup allows in-situ monitoring of a rotating bilayer of emulsion droplets using an optical microscope. The oil volume fraction in a compressed layer of oil droplets stabilized against coalescence was measured experimentally as a function of time for different radial accelerations. The sedimentation was simulated using CFD in order to test the applicability of the computational method and the Ishii-Zuber drag law for very high dispersed phase volume fractions. Quantitative agreement of emulsion sedimentation as a function of time between the experiments and simulations is good at higher accelerations, but decreases with decreasing accelerations. Coalescence in a centrifuged emulsion, which was destabilized prior to centrifugation by adding sodium chloride, was also quantified. The growth of a pure oil phase on top of the droplet layer was measured as a function of time. From the growth rate, a characteristic time for droplet coalescence with the pure oil phase was deduced. The experimental method may serve as a tool to study the compression and coalescence kinetics of emulsions under enhanced gravity, which may be of use to assess emulsion stability for industrial applications. Possible improvements of the current experimental setup are also discussed.

Keywords: emulsion, compression, coalescence, microscopy, droplet, CFD, drag law.
\end{abstract}




\section{Introduction}

The behavior of dense emulsions in flow fields with radial accelerations is of major importance for separation processes using cyclonic devices or centrifuges. Major examples are separation of crude oil and water during oil production, and milk processing in the food industry. The design of these separation processes is often strongly based on the results of Computational Fluid Dynamics, which has become an important tool for the process industry in recent years. For reliable modeling, information about the sedimentation rate, and kinetics of coalescence and breakup of droplets in a dense emulsion under enhanced gravity is needed.

A key parameter for modeling is the choice of the drag law for droplets as it will impact the calculated rate of sedimentation. For dense droplet-laden flows, the drag law of Ishii and Zuber is often used [1]. The Ishii-Zuber drag law is an extension of the well known Schiller-Naumann drag law [2]. It can be applied to flows with higher volume fractions of the disperse phase by incorporating hindered settling. As a droplet moves through the fluid it will induce a motion of the continuous phase and thereby deform the surrounding fluid. When other droplets are present in this surrounding fluid they will be subjected to this deformation as well. Due the Laplace pressure, the surrounding droplets will resist deformation more than the continuous fluid, leading to a higher local viscosity acting on the moving droplet. Therefore, the drag for these dense systems is modeled by assuming similarity to the single droplet case and using an expression for the mixture viscosity instead of the continuous phase viscosity. Besides the Ishii-Zuber drag law, other drag laws have been proposed for dense systems [3]. Still, the Ishii-Zuber law is a popular choice for CFD since it can be applied for a wide range of multiphase systems and flow parameters.

In addition to applying proper relation for the drag law, a correct prediction of the droplet size distribution and its effects on the flow field will increase the accuracy of multiphase flow CFD. In multiphase CFD, droplet breakup and coalescence kinetics can be taken into account using the approach of population balance equations (PBE) [4]. Analytical hydrodynamic models to predict the timescale of coalescence between two droplets have been proposed, which cannot generally be applied to dense flowing emulsions under enhanced gravity, however. Apart from analytical models, input for the coalescence functions in PBE modeling can also be provided by experiments, in principle. For oil/water separation applications, the kinetics of coalescence between droplets will impact the rate of separation. The mean droplet size will increase in the system when coalescence dominates over breakup, which will then accelerate the separation.

The dynamics of coalescence in centrifuged emulsions have been recently investigated by us using a tabletop centrifuge [5]. From the experiments, we derived characteristic coalescence times of the droplets in the emulsion as a function of the radial acceleration and surfactant concentration. Analysis of the samples was performed ex-situ, however, which increased the experimental error and made the experiments time-consuming. 
In this paper, we present a study of compression and coalescence in an emulsion under enhanced gravity using a custom-built centrifuge, which allows in-situ microscopic observation of the emulsion during centrifugation. The degree of compression in a stable emulsion is expressed as the oil volume fraction in the compressed droplet layer. We compare the experimental results with CFD calculations to test the applicability of the computational method and the IshiiZuber drag law to high dispersed phase fractions. Further, we measure the rate of coalescence in an unstable emulsion as a function of the radial acceleration. From the coalescence experiments, characteristic times for coalescence of a droplet with a pure oil phase under enhanced gravity can be derived.

\section{Experimental and computational methods}

\subsection{Chemicals}

Sodium n-dodecyl sulfate (SDS, ACS reagent, $\geq 99 \%$ ) and sodium chloride ( $\mathrm{NaCl}$, ACS reagent, $\geq 99 \%$ ) were purchased from Sigma Aldrich. Sylgard 184 Silicone Elastomer kit was purchased from Dow Corning. As dispersed phase Sil180 silicone oil (Dow Corning) was used. At 293 K, the density of Sil180 is $931 \mathrm{~kg} \mathrm{~m}^{-3}$ and the viscosity $10.4 \mathrm{mPa}$ s. Aqueous solutions of $\mathrm{NaCl}$ (5 wt\%) and SDS $(10 \mathrm{mM})$ were prepared with Millipore water. The density of the $5 \mathrm{wt} \%$ aqueous $\mathrm{NaCl}$ solution was $1032 \mathrm{~kg} \mathrm{~m}^{-3}$ at $293 \mathrm{~K}$. The density difference between pure water and the oil is $\Delta \rho=69 \mathrm{~kg} \mathrm{~m}^{-3}$, and for the salt solution and the oil $\Delta \rho=101 \mathrm{~kg} \mathrm{~m}^{-3}$.

\subsection{Microcentrifuge experiments}

For the microcentrifuge experiments, a custom-made setup was used. Figure 1A displays a photo of the experimental setup. The setup consists of a disk, which is connected to a DC motor. The range of accessible rotation frequencies $f$ is $1.5 \mathrm{~Hz}<f<100 \mathrm{~Hz}$. A sample holder can be mounted on the disk. The distance $r$ between the center of the sample holder and the motor axis is $10 \mathrm{~cm}$. The radial acceleration $a$ is given by $a=(2 \pi f)^{2} r$. The range of accelerations is thus $3.95 \mathrm{~m} \mathrm{~s}^{-2}<a<39500 \mathrm{~m} \mathrm{~s}^{-2}$, or $0.402<a / g<4020$, if expressed in multiples $a / g$ of the normal gravitational acceleration $g=9.81 \mathrm{~m} \mathrm{~s}^{-2}$. For the entire range of $a / g$ the induction time of the motor to reach $95 \%$ of the desired acceleration was $<0.5 \mathrm{~s}$. The disk and the motor are enclosed in a protective housing. The entire assembly is placed on a custom-made plate, which is mounted on an inverted optical microscope (Axiovert 200, Zeiss). Three set screws allow to align the sample with the objective of the microscope. A high-speed camera (Y4-S2, IDT Inc.) is connected to the microscope. A software trigger was used (Motion Pro Studio, IDT Inc), which triggered recording when at least one pixel in the image was not black, indicating that the sample chamber, which is transparent, is passing by the microscope. A frame rate of $5000 \mathrm{~Hz}$ and an exposure time of $100 \mathrm{~ns}$ were 

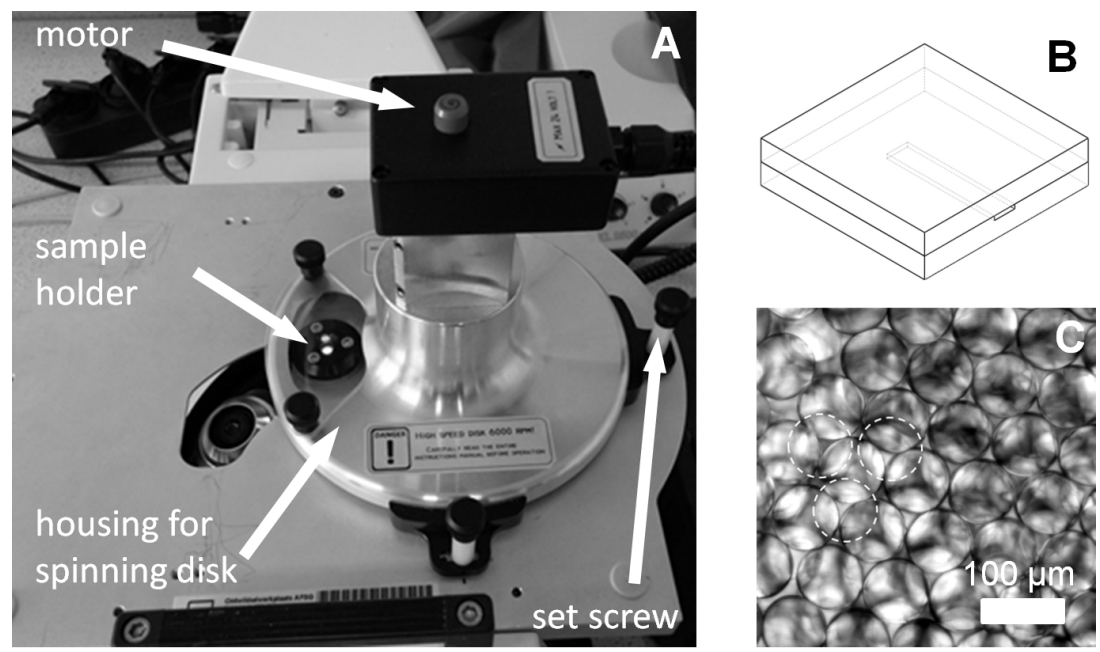

Figure 1: Panel A: photograph of the experimental setup. Panel B: sketch of the sample chamber used for the centrifuge experiments. The chamber is made from two PDMS disks. The bottom disk contains a channel of dimensions $5.25 \times 1 \times 0.2 \mathrm{~mm}^{-3}$, where the emulsion is inserted. Panel C: photograph of a oil-in-water emulsion in the sample chamber. The oil phase was Sil180 silicone oil, the aqueous phase a $10 \mathrm{mM}$ SDS solution. The top layer of a bilayer of monodisperse droplets with a mean diameter $79.3 \mu \mathrm{m}$ is displayed. The position of the second droplet layer is indicated by the dotted circles.

used for the experiments. The recorded images were processed with the program ImageJ using custom-written scripts.

A drawing of the sample chamber, which is placed in the sample holder, is displayed in Figure 1B. The sample chamber consists of two parts, which are made from polydimethylsiloxane (PDMS). The bottom part contains a channel (dimensions $5.25 \times 1 \times 0.2 \mathrm{~mm}^{3}$ ), which is open to one side. The top part of the sample holder contains no features. The parts are made from mold casting using the Sylgard Elastomer Kit, which consists of liquid PDMS and a curing agent [6]. The top and bottom part are chemically bonded together after exposure to an oxygen plasma. Before each experiment, the sample chamber was exposed to an oxygen plasma to render the chamber walls hydrophilic, in order to enable wetting of the channel walls with the continuous phase [6].

Monodisperse droplets of Sil180 silicone oil in a $10 \mathrm{mM}$ aqueous SDS solution were produced with a microfluidic T-junction. The experimental setup and parameters have been described previously [7]. An oil-in-water emulsion with a mean droplet diameter $d=(79.3 \pm 0.8) \mu \mathrm{m}$ was obtained. The emulsion was stable for the entire duration $(\sim 4$ weeks) of the experimental work. The emulsion creamed in the storage flask; droplets were removed from the dense layer using a pipette and injected into the sample chamber. The mean oil volume fraction $\phi_{0}$ 
in the creamed emulsion layer was measured by removing $1 \mu \mathrm{l}$ of sample and counting the number of droplets after spreading of the test volume on a microscope slide. From ten measurements $\phi_{0}=0.72 \pm 0.03$ was obtained. Figure $1 \mathrm{C}$ displays a photograph of the emulsion in the sample chamber. The droplets have a circular cross section and form a bilayer. In the image shown, the image was focussed on the top layer of droplets. The second layer of droplets is visible only diffuse, the position of the droplets relative to the first is sketched by the dotted circles in Figure 1C.

\subsection{Numerical calculations}

$$
C_{D}=\frac{24}{R e_{m}}\left(1+0.15 R e_{m}^{0.687}\right)
$$

The Reynolds number based on the mixture viscosity is defined as $R e_{m}=\rho_{w}\left|\mathbf{u}_{\mathbf{o}}-\mathbf{u}_{\mathbf{w}}\right| d / \eta_{m}$. Finally, the mixture viscosity is given by

$$
\eta_{m}=\eta_{w}(1-\phi)^{-2.5 \frac{\eta_{o}+0.4 \eta_{w}}{\eta_{o}+\eta_{w}}}
$$

Here $\eta_{o}$ and $\eta_{w}$ are the dynamic viscosities of the oil and water respectively.

The governing equations were solved using the commercial CFD package Ansys CFX 13.0. A mesh of 3.3 million hexahedral elements and the use of double precision executables are required to capture the steep gradients in volume fraction and to avoid strong oscillations near that gradient in the numerical solution. A no slip condition is imposed on the walls. The use of a spatially varying centrifugal force lead to numerical oscillations in volume fraction. Therefore, a constant body force is used here. Due the large radius at which the chamber is placed compared to its dimensions of the error made here is smaller than $1 \%$. All other parameters used in the calculation, such as $d, a$ and $\phi_{0}$ are matched with the experimental values. As initial condition a homogeneous emulsion with $\phi_{0}$ is used.

\section{Results and discussion}

\subsection{Emulsion compression}

Figure 2A displays a contour plot of the oil volume fraction $\phi$ in the sample chamber during centrifugation obtained from the CFD calculations at $103 \mathrm{~g}$ after $10 \mathrm{~s}$ of centrifugation as an example. Due to the density difference between the two phases $\left(\Delta \rho=69 \mathrm{~kg} \mathrm{~m}^{-3}\right)$, oil is concentrated at the right side of the sample chamber. At the left side of the sample chamber, pure continuous phase is accumulated. The transition between the oil-rich and oil-free layer is sharp, as can be seen from a cross-sectional plot of $\phi$ along the $y$-axis (Figure 2B). In the oil-rich layer, $\phi(y)$ increases with increasing distance from the boundary between the two layers, and eventually reaches unity. From the $\phi(y)$ profiles we calculate an average oil volume fraction $\phi_{c}$ of the oil-rich layer, in order to compare the 

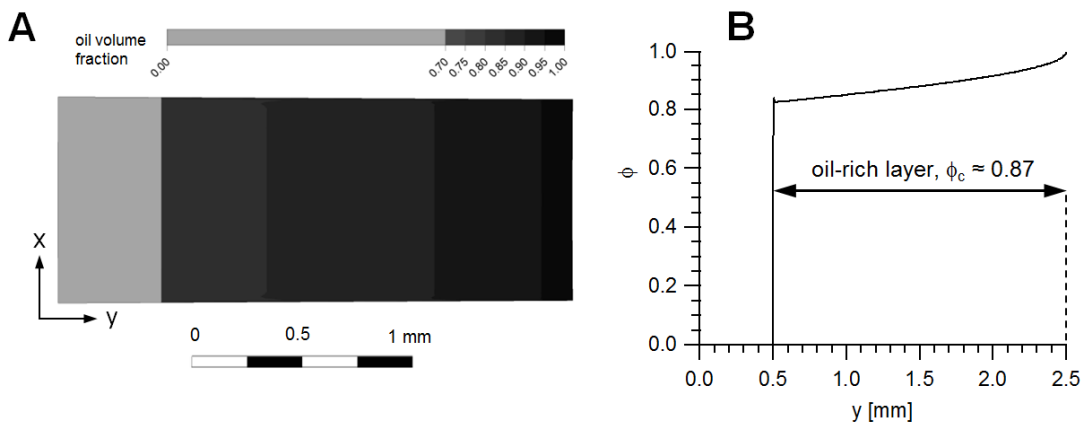

Figure 2: Examples of results of the numerical calculations. Panel A: Contour plot of the oil volume fraction in the sample chamber during centrifugation at $103 \mathrm{~g}, 10 \mathrm{~s}$ after the start of centrifugation. Panel B: cross-sectional profile of the oil volume fraction along the $y$-axis at $x=0.5 \mathrm{~mm}$, extracted from the contour plot in Panel A.

CFD results with the experiments. For the example shown in Figure $2 \mathrm{~B}, \phi_{c}=0.87$ is obtained.

Two images from the compression experiments are shown in Figure 3 as an example. Panel A displays the emulsion at rest before centrifugation. The mean volume fraction of oil is $\phi \approx 0.72$. Panel $\mathrm{B}$ displays a snapshot of the emulsion during centrifugation at $231 \mathrm{~g}, 5 \mathrm{~s}$ after the start of centrifugation. Upon centrifugation, droplets are accelerated towards the axis of rotation, thereby forming a dense layer. From the height of the compressed droplet layer $h_{c}$ and the total height of the liquid column $h_{t}$ we can calculate the averaged oil volume fraction $\phi_{c}$ in the compressed layer, $\phi_{c}=\phi_{0} h_{t} / h_{c}$. For the example shown in Figure $3 \mathrm{~B}$, we obtain $\phi_{c} \approx 0.83$. Figure 3 displays $\phi_{c}$ as a function of time, obtained from the experiments and CFD calculations, for three different radial accelerations. The experimentally measured $\phi_{c}(t)$ increase with time, but eventually approach asymptotic values. For a given time, $\phi_{c}$ increases with increasing acceleration. The maximum volume fraction for hard spheres is $\approx 0.74$, which is close to the experimentally measured value of the monodisperse dense droplet layer from where the emulsion was sampled. For droplets, higher packing fractions can be achieved, as droplets are deformable. The Bond number $B o=\Delta \rho g d^{2} / \sigma$ compares the magnitude of body forces and interfacial tension forces. For our system, at the smallest acceleration of $103 \mathrm{~g}$, we obtain $B o \approx 0.11$, which indicates that droplet deformation will take place [9], and that the measured values of $\phi_{c}>0.72$ are physically reasonable. The maximum oil volume fractions at the corresponding $B o$ are: $\phi_{c, \text { max }}(B o=0.11)=0.75, \phi_{c . \max }(B o=0.25)=0.79$ and $\phi_{c, \max }(B o=0.55)$ $=0.90$. The $\phi_{c}(t)$ curves obtained from the CFD calculation also increase with increasing $\mathrm{t}$, and $\phi_{c}(t)$ also increases with acceleration for a given $t$. The curves do not approach an asymptotic value, however, and for $103 g$ and $231 g$ also differ quantitatively from the experimental data. 

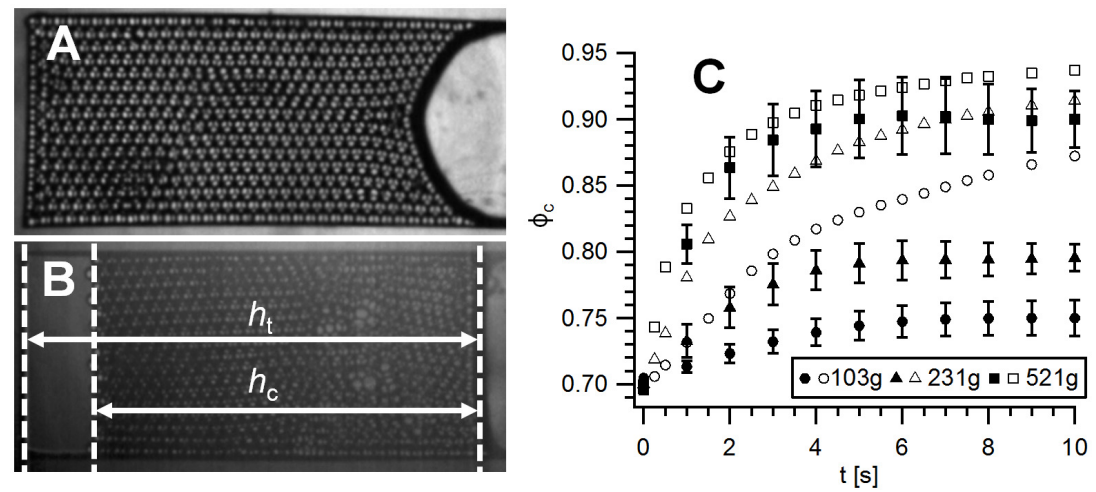

Figure 3: Panel A: photograph of the emulsion at rest. The droplet diameter is $79.3 \mu \mathrm{m}$, the oil volume fraction is 0.72 . Panel B: Photograph of the same emulsion as in panel A after $5 \mathrm{~s}$ of centrifugation at $231 \mathrm{~g}$. $h_{c}$ and $h_{t}$ indicate the height of the compressed emulsion layer and total height of the liquid column, respectively. Panel C: mean oil volume fraction $\phi_{c}$ in the compressed droplet layer as a function of time for different accelerations. Filled symbols are the results from microcentrifuge experiments. Each data point is the average of 4 individual measurements. The error bars are $95 \%$ confidence intervals. Open symbols are the results from the CFD calculations.

The agreement between the $\phi_{c}(t)$ curves which were experimentally measured and calculated from CFD is not good, both from a quantitative and qualitative point of view. Generally, the calculated values overestimate the experimental values. One reason for the discrepancy is the fact that in the computational model the oil volume fraction will eventually approach unity for any radial acceleration, as the dispersed phase is not treated as discrete particles, but as a continuous medium. This is a direct consequence of the averaging procedure carried out in the derivation of the two-fluid model. In the experiments, on the other hand, there will be a maximum value of the oil volume fraction for a given $B o$, for each radial acceleration. The applicability of the computational method for high dispersed phase fractions may be enhanced by imposing a maximum dispersed phase fraction $\phi_{\max }$ that can be reached in a given volume element for the CFD calculations.

In addition, the expression for the mixture viscosity was derived for a dilute suspension of liquid spherical droplets by Taylor [10]. Later, Roscoe derived an expression for the mixture viscosity for solid spheres at higher volume fraction [11]. The two expressions are combined in the mixture viscosity in the Ishii-Zuber drag law. However, Taylor assumes spherical droplets, while in the present experiments considerable deformations are seen. Furthermore, while some deviations from the spherical shape are permitted according to Roscoe, his derivations of the mixture viscosity assumes a very wide particle size distribution. 
Here, a monodisperse emulsion is used. These deviations in the experiments from the assumptions in the model derivation also contribute to the observed discrepancies in the results.

\subsection{Emulsion coalescence}

The kinetics of emulsion breakup were investigated with the same system that was used for the compression experiments. Oil-in-water emulsions stabilized by $10 \mathrm{mM}$ SDS are highly stable due to the colloidal forces that prevent droplets from coalescing [12]. The addition of inorganic electrolytes is known to promote coalescence in emulsions stabilized by ionic surfactants such as SDS, due to a decrease of the electric double layer repulsion between the droplet interfaces [12].

One drop of emulsion with a volume of $350 \mathrm{nl}$ and $\phi=0.72$ was added to the sample chamber. The sample was then briefly centrifuged to push all liquid to the bottom of the chamber. Then, a droplet of a $5 \mathrm{wt} \% \mathrm{NaCl}$ aqueous solution (350 $\mathrm{nl})$ was added to the sample chamber. A snapshot of the sample chamber after addition of the two solutions is displayed in Figure 4 (photo at the left). The concentration of SDS after addition of the $\mathrm{NaCl}$ solution changed to $5 \mathrm{mM}$ and the concentration of $\mathrm{NaCl}$ to $2.5 \mathrm{wt} \%$. The interfacial tension of Sil180 and an aqueous phase with this composition was measured to be $5.4 \mathrm{mN} \mathrm{m}^{-1}$. Emulsions prepared in this way were centrifuged for 10 minutes at different accelerations in the range 64 $<a / g<2260$. Figure 4 displays snapshots of an emulsion centrifuged at $864 g$ at different times. The droplets are compressed at the top of the emulsion. A layer of pure oil, which grows with time, is visible after 1 minute of centrifugation. As continuous phase is flowing from top to bottom in the emulsion due to the enhanced gravitational acceleration, the film of continuous phase between droplets is always thinnest in the top layer of the emulsion. A thinner film is more likely to rupture, hence coalescence takes place mostly in the top layer of the emulsion. In addition to coalescence between oil droplets and the pure oil homophase, coalescence between droplets in the emulsion layer was also observed. This is best visible in the snapshot in Figure 4 taken after 10 minutes. The extent of drop-drop coalescence was not evaluated in this work, as the presence of a droplet bilayer causes problems for automated image analysis.

The volume fraction of coalesced oil $x_{c}=V_{c} / V_{t}$ was evaluated. $V_{c}$ and $V_{t}$ are the volumes of coalesced oil and total volumes of oil in the system, respectively. Figure 5A displays three curves $x_{c}(t)$ as examples. Curve 1 was recorded at $2260 g$ acceleration, curves 2 and 3 were recorded at $1282 g$ acceleration. For curve $1, x_{c}(t)$ increases approximately linearly until $x_{c}=1$ is reached, which indicates that all oil from the emulsion has coalesced. From a linear fit to the data points for which $x_{c}<1$ we calculate a mean coalescence rate $r_{c}=d x_{c} / d t$. For curve 2, $x_{c}<1$ for the duration of the experiment. The difference in slopes between curves 1 and 2 indicates that the coalescence rate is larger for $2260 \mathrm{~g}$ acceleration as compared to $1282 \mathrm{~g}$. It was often observed that coalescence took place before centrifugation was started, as a few seconds passed between addition of the $\mathrm{NaCl}$ solution and the start of centrifugation. Curve 3 in Figure 5A displays an example where significant coalescence occurred before centrifugation and thus the intercept of the linear fit 

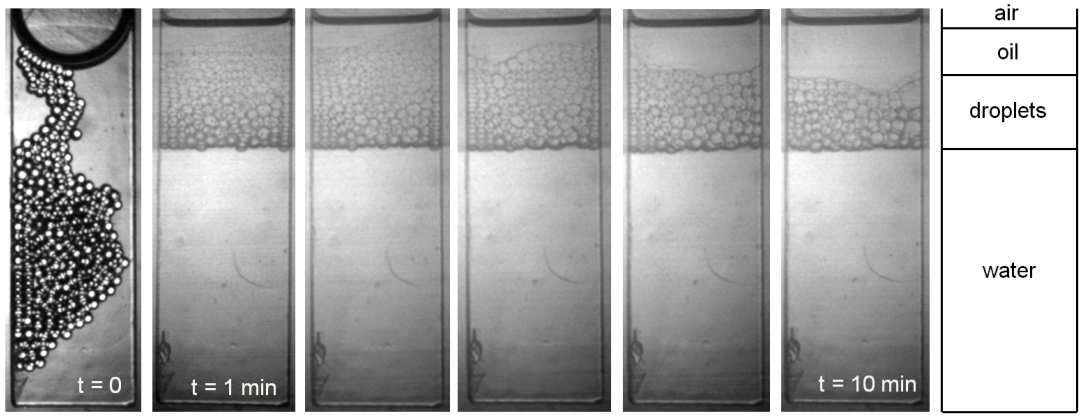

Figure 4: Snapshots of an emulsion after addition of a $5 \mathrm{wt} \% \mathrm{NaCl}$ aqueous solution during centrifugation at $864 g$ centrifugal acceleration. The picture at the left displays the sample chamber after additions of $350 \mathrm{nl}$ emulsion and $350 \mathrm{nl} \mathrm{NaCl}$ solution. The other photos display the sample chamber at different times of centrifugation. The sketch at the right schematically displays the distribution of phases during centrifugation.

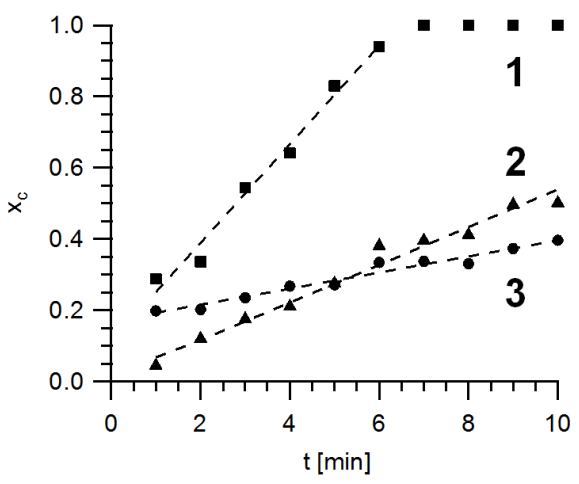

Figure 5: Relative volume fraction of coalesced oil $x_{c}$ as a function of centrifugation time for three individual experiments as examples. The dotted lines are linear fits to the data points. The curves are discussed in detail in section 3.2 .

$x_{c}(t)$ at $t=0$ would deviate strongly from zero. The first data point was taken after one minute centrifugation, $x_{c}(t)$ increased approximately linear from this point on.

The slopes of curves 2 and 3 differ significantly from each other, even though experiments were performed at the same acceleration. The scatter of the data points was generally large, which is visualized in Figure 6A displaying $r_{c}$ as a function of the relative radial acceleration $a / g$ obtained from individual measurements. The reason for this large scatter is currently not known. For each value $a / g$, 5-7 individual measurements were performed and the average value $\left\langle r_{c}\right\rangle$ was calculated. Even though the error bars in Figure 6A are large, $\left\langle r_{c}\right\rangle$ increases 

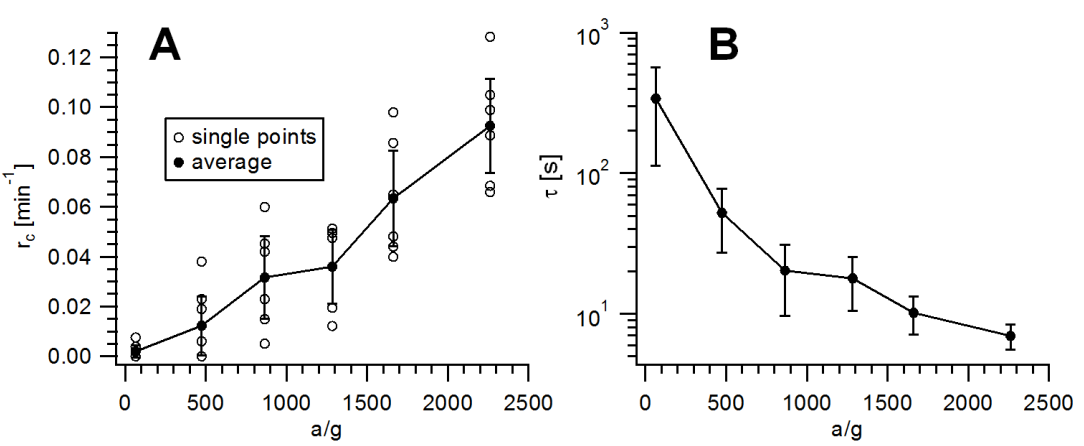

Figure 6: Mean relative coalescence rate $r_{c}$ (panel A) and mean coalescence time $\tau$ (panel B) between a droplet and the pure oil phase as a function of the relative acceleration $a / g$. The open circles in Panel $\mathrm{A}$ are results of individual measurements, the filled circles are average data points from 5-7 measurements. Error bars are 95\% confidence intervals.

approximately linearly with increasing $a / g$. The coalescence rate $r_{c}$ is not a universal property, as it depends on the area of the pure oil/emulsion interface $A_{i}$. A more general parameter is the characteristic coalescence time $\tau$, which is the time it takes for the thin film of continuous phase between the droplet and the pure oil phase to drain and rupture. We have shown previously that $\tau$ can be calculated as $\tau=A_{i} \phi_{t} / 4 V_{0} q_{t} r_{c}$ [5]. $A_{i}$ is given by $A_{i}=h w$, where $h$ and $w$ are the channel height and width, respectively. $\phi_{t}$ is the dispersed phase volume fraction in the top layer of the emulsion, for which we will assume $\phi_{t}=1 . V_{0}$ is the total volume of oil in the system, $q_{t}$ is the ratio of cross-sectional area $A_{d}$ and volume $V_{d}$ of a droplet in the top layer of the emulsion, $q_{t}=A_{d} / V_{d}$.

From the images we see that the droplets in the top layer are deformed. If we assume the droplets to be in the shape of a rhombic dodecahedron, which is a common approximation for deformed droplets in a dense emulsion layer, then we obtain $q_{t} \approx 2.29 / d$, where $d$ is the diameter of an undeformed droplet of the same volume. The coalescence time $\tau$ is displayed in Figure 6B as a function of the relative radial acceleration $a / g ; \tau$ decreases with increasing $a / g$.

In the simplest model for film drainage, a circular film forms at the droplet interface which is parallel with the pure oil interface. The coalescence time $\tau$ is then given by: $\tau=12 \pi \eta_{c} r_{f}^{4} / F h_{c}^{2}$ [13]. $\eta_{c}$ is the continuous phase viscosity, $r_{f}$ the film radius, $F$ the compressive force and $h_{c}$ the critical film thickness, at which coalescence will occur. The equation predicts a decrease of $\tau$ with increasing compressive force, which was observed in our experiments.

Several factors will cause quantitative deviation of the experimental data from this simple model, however. Firstly, the film radius itself will depend on the compressive force, an estimate is given by $r_{f}^{2}=F r_{d} / 2 \pi \sigma$, where $r_{d}$ is the droplet radius and $\sigma$ the interfacial tension [12]. Due to the confinement of droplets in a dense emulsions, $r_{f}$ cannot increase arbitrarily with $F$, but will take on a maximal 
value at a certain force. Secondly, the critical film thickness is mostly only known as an order-of-magnitude estimate, and is thus a considerable source of error, as $\tau \propto h^{-2}$. Thirdly, as the coalescing droplet is surrounded by a dense emulsion, the flow of continuous phase may be significantly impeded due to the high hydraulic resistance of the network of channels of continuous phase that is formed between the emulsion droplets, as compared to film drainage between an isolated drop and the pure oil interface. Fourthly, the experiments were performed with a micellar solution as the continuous phase. The presence of micelles may further modulate the interaction force between the droplets at distances $<100 \mathrm{~nm}$ [12], which is difficult to quantify. Hence, a quantitative description of the coalescence dynamics cannot be done in a reliable manner, and we restrict ourselves to the qualitative discussion given above.

\section{Conclusions}

We investigated the sedimentation and coalescence of monodisperse emulsions under enhanced gravity using in-situ microscopy. The novelty of the experimental approach is that it allows direct optical monitoring of emulsion droplets during centrifugation at high $g$-forces.

The agreement between the emulsion compression experiments and numerical simulations was not good for two of the three employed radial accelerations. Possible reasons for this are the loss of interfacial physics due to the averaging procedure used for the two-fluid model and also the disagreement of the experiments with some of the modeling assumptions. We would like to point out that the results demonstrate that the Ishii-Zuber drag law, which is very popular, should be employed critically in CFD calculations simulating multiphase systems with high dispersed phase fractions and/or under conditions where enhanced gravity would cause significant droplet deformation.

In the coalescence experiments it was found that the coalescence rate increased approximately linearly with $a / g$. This behavior was explained qualitatively with a simple film drainage model.

Improvements of the current experimental setup are possible for future work. In order to study coalescence between droplets in the emulsion, a monolayer of droplets must be produced to allow for a better visibility of individual droplets. As we want to work with droplet diameters $<100 \mu \mathrm{m}$ because of their relevance for industrial applications, the height of the sample chamber needs to be $\leq 100$ $\mu \mathrm{m}$. When using PDMS as chamber material, we observed that channels of height $\leq 100 \mu \mathrm{m}$ and width $=1 \mathrm{~mm}$ collapsed due to downward bending of the lid. To avoid this, the sample chamber can be made from glass. The procedure of destabilizing the emulsion by addition of salt may cause coalescence before the start of centrifugation. The destabilization can be triggered in a more reproducible way by choosing a photo-destructible surfactant, that will lose its emulsifying capability upon irradiation of the sample with light [14]. This is expected to reduce the statistical uncertainty of the measured coalescence rate. 


\section{Acknowledgements}

This work is carried out as part of a project of the Institute for Sustainable Process Technology: development of an $\Omega^{2} R$ separator focusing on oil/water separation (project number OG 00-04). We thank Laurens van Campen from Delft University of Technology, Johanna Bos from Frames and Paul Verbeek from FMC Technologies for helpful discussions.

\section{References}

[1] Ishii, M. \& Zuber, N., Drag coefficient and relative velocity in bubbly, droplet or particulate flows. AIChE Journal, 25, pp. 843-855, 1979.

[2] Schiller, L. \& Naumann, A., Uber die grundlegenden Berechnungen bei der Schwerkraftaufbereitung. Zeitschrift des Vereines Deutscher Ingenieure, 77, p. 318, 1933.

[3] Rusche, H., Computational fluid dynamics of dispersed two-phase flows at high phase fractions. Ph.D. thesis, Imperial College of Science, Technology \& Medicine, Department of Mechanical Engineering, London, UK, 2002.

[4] Jakobsen, H., Chemical Reactor Modeling: Multiphase Reactive Flows. Springer, 1st edition, 2008.

[5] Krebs, T., Schroen, C. \& Boom, R., Separation kinetics of an oil-in-water emulsion under enhanced gravity. Chemical Engineering Science, 72, pp. 118-125, 2012.

[6] Qin, D., Xia, Y. \& Whitesides, G., Soft lithography for micro- and nanoscale patterning. Nature Protocols, 5, pp. 491-502, 2010.

[7] Krebs, T., Schroen, C. \& Boom, R., A microfluidic method to study demulsification kinetics. Lab on a Chip, 2012. doi:10.1039/C2LC20930F.

[8] Drew, D., Mathematical modeling of two-phase flow. Annual Review of Fluid Mechanics, 15, pp. 261-291, 1983.

[9] Griggs, A., Zinchenko, A. \& Davis, R., Gravity-driven motion of a deformable drop or bubble near an inclined plane at low Reynolds number. International Journal of Multiphase Flow, 34, pp. 408-418, 2008.

[10] Taylor, G., The viscosity of a fluid containing small drops of another fluid. Proceedings of the Royal Society of London, Series A, 138, pp. 41-48, 1932.

[11] Roscoe, R., The viscosity of suspensions of rigid spheres. British Journal of Applied Physics, 3, pp. 267-279, 1952.

[12] Birdi, K., Handbook of Surface and Colloid Chemistry. CRC Press, 2nd edition, 2003.

[13] Chesters, A., The modelling of coalescence processes in fluid-liquid dispersions: A review of current understanding. Trans IChemE, 69A, pp. 259-270, 1991.

[14] Eastoe, J., Photo-destructible surfactants in microemulsions. Progress in Colloid and Polymer Science, 133, pp. 106-110, 2006. 